\title{
Dispersão por aves de Psidium guajava L. (Myrtaceae) em ambiente ripário na bacia do rio Paraná, Brasil
}

\section{Dispersal by birds of Psidium guajava L. (Myrtaceae) in a riparian environment, Parana river basin, Brazil}

\author{
João Carlos Barbosa da Silva'; José Flávio Cândido Junior²; Huilquer Francisco \\ Vogel $^{3}$; João Batista Campos ${ }^{4}$
}

\begin{abstract}
Resumo
A dispersão da goiabeira (Psidium guajava L.) em ecossistemas naturais brasileiros é um processo crescente e requer estudos sobre a dinâmica desta espécie. Deste modo, os objetivos foram: a) identificar quais espécies de aves se alimentam dos frutos de Psidium guajava; b) descrever as principais táticas de forrageamento das aves, que podem maximizar ou reduzir o sucesso de dispersão da goiabeira. Foram realizadas 47 horas de observação focal de aves consumindo frutos de P. guajava, onde foram registradas 11 espécies de aves (Aratinga leucophthalma, A. aurea, Amazona aestiva, Melanerpes flavifrons, Pitangus sulphuratus, Cyclarhis gujanensis, Turdus leucomelas, Mimus saturninus, Tangara palmarum, T. sayaca e Euphonia chlorotica). As guildas de aves mais representativas foram os onívoros $(54,5 \%)$, seguida dos frugívoros $(27,3 \%)$ e das espécies consideradas insetívoras $(18,2 \%)$. Com exceção dos psitacídeos, que apresentam comportamentos alimentares que normalmente promovem danos à semente, as outras espécies apresentaram comportamentos que as indicam como potenciais dispersores da goiabeira. As aves observadas frequentam ambiente florestal e também áreas abertas, o que potencializa a dispersão da espécie pela maioria dos ambientes, podendo alterar a estrutura e composição específica da vegetação da região. Com aves atuando como dispersores de suas sementes, a colonização de novas áreas pela $P$. guajava é eminente, podendo assim dominar áreas de regeneração ambiental, tornando-se uma espécie danosa a processos ecossistêmicos.

Palavras-chave: Ecologia alimentar. Frugivoria.Goiabeira. Invasão biológica.
\end{abstract}

\begin{abstract}
The dispersion of guava (Psidium guajava L.) in Brazilian natural ecosystems is a growing process and requires studies on its ecological dynamics. Thus, our goals were: a) to identify birds feeding on the fruits of Psidium guajava; b) evaluate the potential success of guava dispersal based in the main
\end{abstract}

\footnotetext{
${ }^{1}$ Universidade Estadual de Maringá, Avenida Colombo, 5790, Zona 7, 97.020-900 Maringá- PR, Brasil. E-mail: jc.ornito@gmail. com (contato principal para correspondência).

${ }^{2}$ Laboratório de Zoologia de Vertebrados e Biologia da Conservação, CCBS - UNIOESTE, rua universitária, 2069, Jd. Universitário - CEP 85814-110, Cascavel, PR, Brasil. E-mail: jflavio@unioeste.br

${ }^{3}$ Universidade Estadual de Maringá, Avenida Colombo, 5790, Zona 7, 97.020-900 Maringá- PR. Licenciado em Geografia e Ciências Biológicas; Doutorando em Ciências Ambientais no Programa de Pós-Graduação da Universidade Estadual de Maringá, UEM. E-mail: huilquer@hotmail.com

${ }^{4}$ Secretaria de Estado de Meio Ambiente e Recursos Hídricos - SEMA, rua Desembargador Motta, 3384 - CEP 80430-200, Curitiba, PR. E-mail: joaobatista@iap.pr.gov.br
} 
local bird foraging tactics. We performed 47 hours of focal observation of birds feeding in P. guajava and we recorded 11 bird species (Aratinga leucophthalma, A. aurea, Amazona aestiva, Melanerpes flavifrons, Pitangus sulphuratus, Cyclarhis gujanensis, Turdus leucomelas, Mimus saturninus, Tangara palmarum, T. sayaca and Euphonia chlorotica). The most representative bird guilds were omnivores $(54.5 \%)$, followed by frugivores $(27.3 \%)$ and insectivorous $(18.2 \%)$. With the exception of parrots which eating behavior normally promote damage to the seed, the other species have behaviors that characterize them as potential guava dispersers. The birds observed explore forest environment and also open areas, which enhances dispersion of guava to many environments, altering the regional structure and composition of vegetation. As birds acting as dispersers of seeds of guava, the colonization of new areas by P. guajava is imminent. This species can dominate areas of environmental regeneration, becoming a damaging species to natural ecosystem processes.

Keywords: Feeding ecology. Frugivory. Guava tree. Biological invasion.

\section{Introdução}

O consumo de frutos por animais muitas vezes representa a fase inicial da dispersão de plantas com síndrome zoocórica (JORDANO; SCHUPP, 2000). Estima-se que em florestas tropicais mais da metade das plantas sejam dispersas por animais (HOWE; SMALLWOOD, 1982). Deste modo, estudos de frugivoria por aves são fundamentais para a compreensão da dieta e comportamento alimentar das aves consumidoras de frutos e para a compreensão do processo de dispersão de sementes e o estabelecimento de novas populações de espécies vegetais (JESUS; MONTEIRO-FILHO, 2007).

Quando a frugivoria envolve uma planta exótica ou alóctone, essa interação pode estimular a dispersão de seus diásporos e contribuir para que a espécie se torne invasora, principalmente se esta apresentar sementes com tegumentos rígidos e frutos com características atrativas para seus consumidores (RICHARDSON et al., 2000, STAGGEMEIER; GALETTI, 2007).

Uma planta que reúne tais características é a goiabeira (Psidium guajava L.), pertencente à família Myrtaceae. Atualmente, a espécie se distribui ao longo de áreas tropicais e subtropicais do planeta (ORWA et al., 2009). O conhecimento sobre a origem da espécie é incerto e, por isto, é considerada exótica no Brasil, sendo consumida e dispersa principalmente por aves e macacos, embora outros mamíferos, lagartos, peixes e formigas também possam também realizar sua dispersão
(GRESSLER; PIZO; MORELLATO, 2006). Os frutos são carnosos, relativamente macios e ricos em água e carboidratos (LANDRUM; KAWASAKI 1997, PIZO, 2002), o que justifica o consumo por esses animais.

Dentre algumas das características que aumentam o potencial da goiabeira para sua dispersão zoocórica destacam-se alguns, como a possibilidade de mais de um ciclo de produção de frutos ao longo do ano (PEREIRA; ANDRADE, 1994; SOMARRIBA, 1986) e a longa viabilidade das sementes e o seu grande número (até 250) por fruto (GRESSLER; PIZO; MORELLATO, 2006). Além disso, as sementes podem ser consideradas pequenas em comparação com outras Myrtaceae e em comparação com outros itens consumidos por seus dispersores (GRESSLER; PIZO; MORELLATO, 2006; LAKE; LEISHMAN, 2004). Estas sementes apresentam testa (tegumento externo) bastante resistente (LANDRUM; SHARP, 1989), o que minimiza as chances de dano durante o processo de consumo.

A goiabeira foi registrada pela primeira vez na planície de inundação do alto rio Paraná em 1992 (Herbário da Universidade Estadual de Maringá/ Coleção Especial Vegetação Ripária; HUEM 995). Em monitoramentos realizados em áreas de regeneração natural nessa planície foi possível verificar um aumento do IVI (índice de valor de importância) da espécie em relação às demais, com incremento de (91\%) entre os anos de 2002 e 
2007 (ZVIEJKOVSKI, 2008). A goiabeira também foi registrada no banco de sementes da planície de inundação do rio Paraná em diferentes ambientes e profundidades (CAMPOS, SOUZA, 2003) e sua capacidade de dispersão em áreas úmidas foi observada por Nóbrega et al. (2009), demonstrando a plasticidade adaptativa da espécie sob diferentes condições ambientais.

Com base nas considerações apontadas, os objetivos deste trabalho foram: a) identificar quais espécies de aves se alimentam dos frutos de Psidium guajava; b) descrever as principais táticas de forrageamento das aves que podem maximizar ou reduzir o sucesso de dispersão da goiabeira. Tais conhecimentos podem auxiliar na compreensão da dispersão desta espécie em floresta ripária no noroeste do Paraná e em outros ecossistemas onde P. guajava represente potencial invasivo.

\section{Material e Métodos}

Área de Estudo

$\mathrm{O}$ estudo foi desenvolvido na vegetação ripária do córrego Caracu (2245'55”S e 53॰15’30”W), tributário da margem esquerda do rio Paraná, situado no município de Porto Rico, estado do Paraná, Brasil (Figura 1). A vegetação da região é classificada como Floresta Estacional Semidecidual e o clima é do tipo $C f a$ - clima tropical-subtropical com verão quente (média superior a $22^{\circ} \mathrm{C}$ anuais), com precipitação anual média de $1500 \mathrm{~mm}$ (IBGE, 1992). Na vegetação ripária da região ocorrem naturalmente Cecropia pachystachya Trécul, Croton urucurana Baill., Inga vera Willd., Chamaechrista sp., Cereus sp., Cariniana estrellensis (Raddi) Kuntze, Lonchocarpus cultratus (Vell.) A.M.G. Azevedo \& H.C. Lima, Tabernaemontana catharinensis A.DC., Gallesia integrifolia (Spreng.) Harms, Anadenanthera sp., entre outras (CAMPOS; SOUZA, 1997).
Figura 1 - Localização da área de estudo (*) nas margens do córrego Caracu, afluente da $\mathrm{m}$ a r g e $\mathrm{m}$ esquerda do rio Paraná, no município de Porto Rico, estado do Paraná, Brasil.

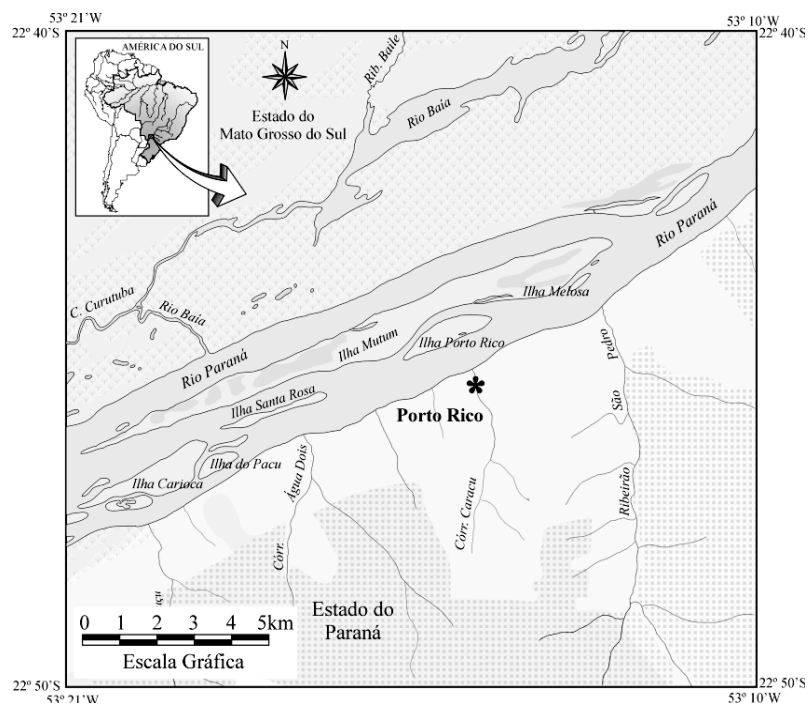

Fonte: Autores

\section{Coleta de Dados}

O estudo foi conduzido observando-se três indivíduos de Psidium guajava, com altura aproximada de $4 \mathrm{~m}$, com grande abundância de frutos, próximos entre si e que se localizavam na borda da vegetação ripária. As observações ocorreram entre as 06:00 - 10:00 h e 16:00 - 19:00 $\mathrm{h}$, totalizando $47 \mathrm{~h}$ de estudo, entre novembro de 2009 e fevereiro de 2010. As amostragens foram realizadas observando aves que consumiam frutos de goiabeira, através da técnica de indivíduo focal, a uma distância média de $20 \mathrm{~m}$ das plantas, utilizando binóculo. Foram consideradas como visitas apenas quando houve o consumo efetivo dos frutos pela espécie de ave. A nomenclatura das espécies está de acordo com o Comitê Brasileiro de Registros Ornitológicos (COMITÊ BRASILEIRO DE REGISTROS ORNITOLÓGICOS, 2011) e a classificação em guildas tróficas das espécies foi baseada em Willis (1979) e Motta-Júnior (1990).

Para as amostragens foi utilizada uma adaptação da metodologia proposta por Pizo (1997), no qual 
foram registradas as espécies de aves consumidoras de frutos, e anotado: i) o número de visitas de cada espécie; e ii) comportamento de forrageamento baseado na forma de obtenção dos frutos ( $\mathrm{I}=$ investir; $\mathrm{P}=$ pular; $\mathrm{C}=$ caminhar; $\mathrm{Pe}=$ pendurar; $\mathrm{R}=$ respigar; $\mathrm{A}=$ alcançar) e também na forma de consumo do recurso $(\mathrm{M}=$ mandibular e $\mathrm{P}=$ picar $)$.

A nomenclatura de forrageamento seguiu Volpato e Mendonça-Lima (2002) no qual: investir é o comportamento designado quando a ave voa de um poleiro para atacar um item alimentar próximo; pular corresponde aos saltos; caminhar são os movimentos realizados linearmente sem voos; pendurar ocorre quando a ave usa as pernas e os dedos para suspender o corpo abaixo do nível dos pés para alcançar o alimento; respigar ocorre quando a ave captura o alimento em um substrato próximo em relação a ave; alcançar ocorre quando a ave estende completamente as pernas e/ou pescoço para capturar o alimento. O comportamento de "mandibular" foi definido a posteriori, uma vez que é uma variação do "esmagar" no qual a ave movimenta partes do fruto entre as maxilas antes de engolir; e por fim "picar" que ocorre quando a ave bica e remove partes do fruto.

\section{Análise dos Dados}

Para testar a possibilidade de ocorrência de um grupo trófico ou espécies com maior propensão à dispersão, foi utilizada análise de variância não paramétrica (Kruskal-Wallis) e o Teste a posteriori de Dunn (SIEGEL; CASTELLAN-JUNIOR, 1988), comparando as diferenças entre número de visitas de aves, em relação às guildas. Foi utilizado o teste $\chi^{2}$ com o objetivo de comparar o número de visitas em relação às espécies de aves. Para testar se as estratégias de forrageamento diferiram entre as guildas tróficas foi efetuada uma análise de correspondência (LEGENDRE; LEGENDRE, 2003). Os eixos interpretados foram obtidos através do critério de broken-stick e posteriormente testados através de análise de variância não paramétrica (Kruskal-Wallis).

\section{Resultados}

Foi registrada a presença de 11 espécies pertencentes a oito famílias se alimentando dos frutos (Tabela 1). No total foram registradas 87 visitas. A guilda de aves mais representativa foi a dos onívoros (54,5\%), seguida dos frugívoros $(27,3 \%)$ e das espécies insetívoras (18,2\%). A comparação do número de visitas de aves em relação às guildas não foi significativamente diferente $(\mathrm{H}=2,00, \mathrm{gl}=2$; $\mathrm{P}>0,05)$. A comparação entre o número de visitas em relação às espécies de aves evidenciou diferença significativa $\left(\chi^{2}=117,95, \mathrm{gl}=10 ; \mathrm{P}<0,01\right)$. Uma única espécie (Tangara sayaca) foi responsável por $40,2 \%$ do total de visitas durante o estudo.

$\mathrm{Na}$ Análise de Correspondência (Figura 2), os eixos 1 e 2 retiveram $37,8 \%$ da variação total, sendo $30 \%$ da variação referente ao eixo 1 e 7,8\% ao eixo 2. Em relação ao eixo 2 da ordenação, não houve separação das espécies $(\mathrm{H}=2,96, \mathrm{gl}=2$; $\mathrm{P}$ $=0,22)$. Cabe ressaltar que não houve separação entre onívoros e insetívoros, representando padrões semelhantes na obtenção e manipulação dos frutos. 
Tabela 1 - Espécies observadas se alimentando dos frutos de Psidium guajava em vegetação ripária, no município de Porto Rico, estado do Paraná, Brasil. Os comportamentos registrados foram categorizados em Estratégia de forrageamento: $\mathrm{I}=$ investir; $\mathrm{Pu}=$ pular; $\mathrm{C}=$ caminhar; $\mathrm{Pe}=$ pendurar; $\mathrm{R}=$ respigar; $\mathrm{A}$ = alcançar; $\mathrm{M}=$ mandibular; $\mathrm{P}=$ picar. Guilda trófica: $\mathrm{F}=$ Frugívora, $\mathrm{O}=$ Onívora e $\mathrm{I}=$ Insetívora. $\mathrm{N}=$ número de visitas, $\mathrm{F}_{\mathrm{o}}=$ Frequência de registros).

\begin{tabular}{|c|c|c|c|c|c|c|c|c|c|c|c|}
\hline \multirow[b]{2}{*}{ Família/espécie } & \multicolumn{2}{|c|}{ Visitas } & \multicolumn{8}{|c|}{$\begin{array}{l}\text { Estratégias de } \\
\text { forrageamento }\end{array}$} & \multirow{2}{*}{$\begin{array}{l}\text { Guildas } \\
\text { tróficas }\end{array}$} \\
\hline & $\mathbf{N}$ & $\mathbf{F}_{0}(\%)$ & $\mathbf{I}$ & $\mathbf{P u}_{\mathbf{u}}$ & $\mathbf{C}$ & $\mathbf{P e}_{\mathrm{e}}$ & $\mathbf{R}$ & $\mathbf{A}$ & $\mathbf{M}$ & $\mathbf{P}$ & \\
\hline \multicolumn{12}{|l|}{ Psittacidae } \\
\hline Aratinga leucophthalma (Statius Muller, & 12 & 13,7 & 7 & 0 & 8 & 3 & 8 & 4 & 12 & 12 & $\mathrm{~F}$ \\
\hline Aratinga aurea (Gmelin, 1788) & 6 & 7,0 & 5 & 0 & 1 & 1 & 4 & 2 & 6 & 6 & $\mathrm{~F}$ \\
\hline Amazona aestiva (Linnaeus, 1758) & 4 & 4,5 & 4 & 0 & 2 & 1 & 4 & 0 & 4 & 4 & $\mathrm{~F}$ \\
\hline \multicolumn{12}{|l|}{ Picidae } \\
\hline Melanerpes flavifrons (Vieillot, 1818) & 3 & 3,5 & 3 & 0 & 0 & 0 & 3 & 0 & 0 & 3 & I \\
\hline \multicolumn{12}{|l|}{ Tyrannidae } \\
\hline Pitangus sulphuratus (Linnaeus, 1766) & 4 & 4,5 & 4 & 3 & 0 & 0 & 1 & 3 & 0 & 4 & $\mathrm{O}$ \\
\hline \multicolumn{12}{|l|}{ Vireonidae } \\
\hline Cyclarhis gujanensis (Gmelin, 1789) & 2 & 2,5 & 2 & 1 & 0 & 0 & 2 & 0 & 0 & 2 & I \\
\hline \multicolumn{12}{|l|}{ Turdidae } \\
\hline Turdus leucomelas Vieillot, 1818 & 12 & 13,7 & 11 & 6 & 0 & 0 & 12 & 0 & 0 & 12 & $\mathrm{O}$ \\
\hline \multicolumn{12}{|l|}{ Mimidae } \\
\hline Mimus saturninus (Lichtenstein, 1823) & 3 & 3,5 & 2 & 2 & 0 & 0 & 3 & 0 & 0 & 3 & $\mathrm{O}$ \\
\hline \multicolumn{12}{|l|}{ Thraupidae } \\
\hline Tangara palmarum (Wied, 1823) & 3 & 3,5 & 3 & 3 & 0 & 0 & 1 & 2 & 0 & 3 & $\mathrm{O}$ \\
\hline Tangara sayaca (Linnaeus, 1766) & 35 & 40,2 & 33 & 7 & 0 & 8 & 30 & 5 & 0 & 35 & $\mathrm{O}$ \\
\hline \multicolumn{12}{|l|}{ Fringillidae } \\
\hline Euphonia chlorotica (Linnaeus, 1766) & 3 & 3,5 & 3 & 3 & 0 & 1 & 3 & 0 & 0 & 3 & $\mathrm{O}$ \\
\hline Total & 87 & 100 & 77 & 25 & 11 & 14 & 71 & 16 & 22 & 87 & \\
\hline
\end{tabular}

Fonte: Autores

Os principais comportamentos que influenciaram nesta distinção estiveram correlacionados positivamente com o eixo 1 da $\mathrm{CA}$, sendo o comportamento de mandibular o fruto $(\mathrm{r}=0,92)$ e o comportamento de caminhar $(r=0,79)$, os principais responsáveis pela segregação entre guildas. Houve separação entre os padrões comportamentais, distinguindo os frugívoros das demais guildas $(\mathrm{H}=6,54, \mathrm{gl}=2 ; \mathrm{P}=0,03)$, demonstrando que os psitacídeos, aves essencialmente frugívoras, desempenharam comportamento distinto dos demais em relação à obtenção dos frutos de $P$. guajava. 
Figura 2 - Análise de correspondência (CA) dos grupos tróficos, em relação ao total de comportamentos observados durante 0 forrageamento em Psidium guajava em vegetação ripária na no município de Porto Rico, estado do Paraná, Brasil, sendo: (r) o valor de correlação dos principais comportamentos que influenciaram na separação entre eixos. As letras representam as táticas de obtenção de recursos, onde $P$ significa pendurar, $C$ caminhar e $M$ mandibular.

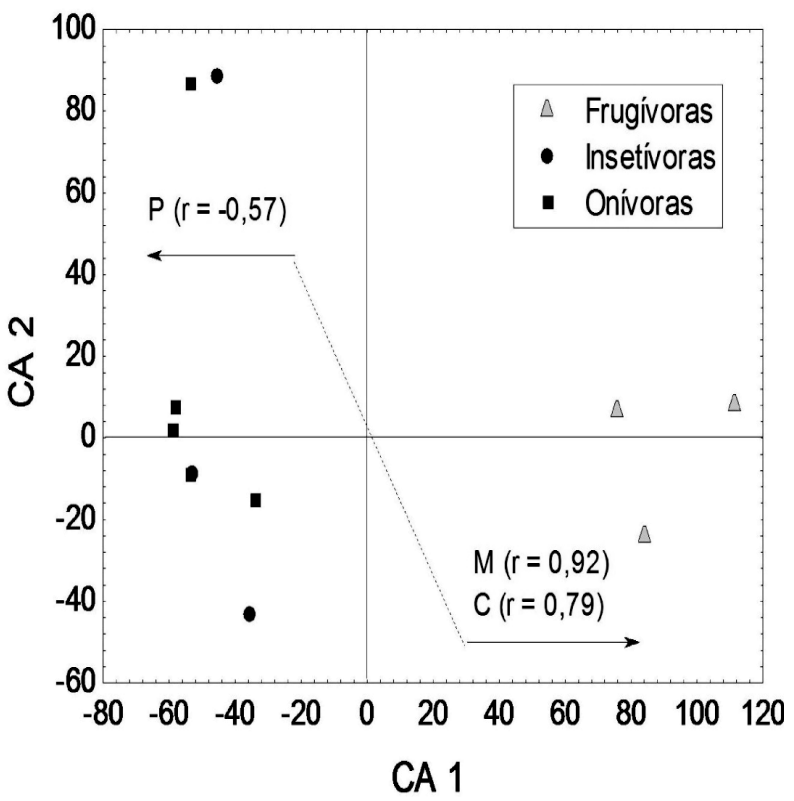

Fonte: Autores

\section{Discussão}

Embora uma estimativa da dispersão de sementes de goiabeira não tenha sido realizada neste estudo, a dieta do dispersor é um bom indicativo de efetividade da dispersão (SCHUPP, 1993). Neste sentido, os melhores candidatos a dispersores em termos de frequência de consumo, manuseio do alimento e tempo de alimentação seriam as aves onívoras registradas, as quais geralmente apresentam relativamente poucas exigências ecológicas quanto ao tipo de vegetação explorada.

A dispersão de $P$. guajava em ambientes fragmentados na parte alta da bacia do rio Paraná pode ter efeitos danosos para outras espécies de planta com dispersão zoocórica. A oferta de seus frutos pode levar à saciedade das espécies consumidoras de frutos da região, prejudicando a dispersão de espécies nativas (CORDEIRO; HOWE, 2003; SILVA; TABARELLI, 2000), o que pode resultar, com o tempo, uma menor diversidade biológica. Essa situação é potencializada no caso da goiabeira, pois seus frutos atraem aves de várias guildas.

As espécies de aves registradas consumindo os seus frutos frequentam áreas florestais e áreas abertas (SICK, 1997; SIGRIST, 2006) e dessa forma podem contribuir para a dispersão da goiabeira em vários ambientes, o que amplifica a capacidade invasora dessa espécie. A pouca exigência em termos de ambiente das espécies de aves registradas é condizente também com sua dieta generalista. Pizo (2004) ressalta a importância de espécies generalistas na dispersão de sementes em áreas fragmentadas ou que sofreram algum tipo de distúrbio. Espécies generalistas estão se tornando mais importantes, uma vez que frequentam habitats que frugívoros especialistas não frequentariam, garantindo assim o movimento de sementes através das paisagens (PIZO, 2004).

Um aspecto relevante é que as relações ecológicas que levam à dispersão de uma dada espécie, nem sempre são muito claras. Como exemplo, pode-se citar que embora Tangara sayaca tenha sido a espécie com maior quantidade de registros de alimentação em P. guajava, para Marcondes-Machado e Rosa (2005), esta ave consome com maior frequência apenas a polpa, não chegando a arrancar os frutos do galho, permitindo que os restos de casca, polpa e sementes caiam próximo à planta-mãe, o que diminuiria as taxas de dispersão. Tal afirmação deve ser interpretada com cautela, pois a interação desta ave com outras espécies vegetais pode ser muito mais efetiva, como evidenciando por Figueiredo et al. (2008) na dispersão de Muntingia calabura L., uma vez que Tangara sayaca possui a capacidade de disseminar sementes pequenas. Por outro lado, a disponibilização de frutos caídos no ambiente por essa ave, permite que estes sejam consumidos 
por organismos terrestres como teiús e ungulados e, deste modo, dispersos de forma secundária (CASTRO; GALETTI, 2004, JULIÁ; PERIS, 2010). Este mecanismo também permite a dispersão por outras aves que podem buscar alimento junto ao solo, como Turdus leucomelas, o que foi observado em algumas ocasiões neste estudo.

Dentre os comportamentos de forrageio registrados, alguns podem afetar o processo de dispersão de sementes, podendo-se citar o "mandibular", realizado pelos psitacídeos. Quando as aves executam esse comportamento, normalmente há perda significativa de sementes e até mesmo sua destruição, de modo que, para muitas espécies de plantas os psitacídeos são caracterizados como predadores e não dispersores de sementes (GALETTI; RODRIGUES, 1992). Entretanto, a resistência das sementes aliada ao reduzido tamanho, pode permitir que ocasionalmente algumas sementes sejam consumidas e dispersas. Além do mais, dentre as aves mencionadas no estudo, os psitacídeos talvez sejam as que mais se deslocam na paisagem e, portanto, os que levariam para mais longe os propágulos da planta.

\section{Conclusões}

Os resultados deste estudo indicam que 11 das espécies de aves silvestres presentes na vegetação ripária do alto rio Paraná se alimentam dos frutos da goiabeira, podendo atuar como dispersores de suas sementes. As aves que se alimentaram dos frutos foram em sua maioria onívoras e com poucas exigências em termos ambientais. Estas espécies percorrem ambientes florestais e áreas abertas, o que intensificaria a dispersão de P. guajava. Com aves atuando como dispersores de suas sementes, a colonização de novas áreas pela espécie é eminente, o que pode levar à competição por agentes dispersores com outras espécies zoocóricas nativas, o que pode alterar a estrutura da vegetação local, principalmente, em locais em processo de recuperação ambiental, como vegetações próximas de cursos hídricos, a exemplo deste estudo.

\section{Agradecimentos}

Agradecemos a Jaime Luiz Lopes Pereira por ceder gentilmente o mapa da área de estudo (Figura 1) que é de sua autoria. Igualmente somos gratos a CNPq (Conselho Nacional de Desenvolvimento Científico e Tecnológico), CAPES (Coordenação de Aperfeiçoamento Pessoal de Nível Superior) pelas bolsas de doutorado de JCBS e HFV, além do Programa de Excelência Acadêmica (PROEX). Ao Programa de Pós-graduação em Ecologia de Ambientes Aquáticos Continentais e ao Nupélia (Núcleo de Pesquisas em Limnologia, Ictiologia e Aquicultura).

\section{Referências}

CAMPOS, J. B.; SOUZA, M. C. Vegetação. In: VAZZOLER, A. E. M.; AGOSTINHO, A. A.; HAHN, N. S. A planície de inundação do alto rio Paraná: aspectos físicos, biológicos e socioeconômicos. Maringá: EDUEM/Nupélia, 1997. p. 331-342.

CAMPOS, J. B.; SOUZA, M. C. Potential for natural forest regeneration from seed bank in an upper Paraná river floodplain, Brazil. Brazilian Archives of Biology and Technology, Curitiba, v. 46, n. 4, p. 625-639, 2003.

CASTRO, E. R.; GALETTI, M. Frugivoria e dispersão de sementes pelo lagarto teiú Tupinambis merianae (Reptilia, Teiidae). Papéis Avulsos de Zoologia, São Paulo, v. 44, n. 6, p. 91-97, 2004.

\section{COMITÊ BRASILEIRO DE REGISTROS} ORNITOLÓGICOS - CBRO. Lista das aves do Brasil. 10. ed. 2011. Disponível em: <http://www. cbro.org.br/CBRO/pdf/AvesBrasil2011.pdf $>$. Acesso em: 27 ago. 2011.

CORDEIRO, N. J.; HOWE, H. F. Forest fragmentation severs mutualism between seed dispersers and an endemic African tree. Proceedings of the National Academy of Science, Washington, v. 100, p. 14052-14056, 2003. 
FIGUEIREDO, R. A.; OLIVEIRA, A. A.; ZACHARIAS, M. A.; BARBOSA, S. M.; PEREIRA, F. F.; CAZELA, G. N.; VIANA, J. P.; CAMARGO, R. A. Reproductive ecology of the exotic tree Muntingia calabura L. (Muntingiaceae) in southeastern Brazil. Revista Árvore, Viçosa, v. 32, n. 6, p. 993-999, 2008.

GALETTI, M.; RODRIGUES, M. Comparative seed predation on pods by parrots in Brazil. Biotropica, Washington, v. 24, n. 2, p. 222-224, 1992.

GRESSLER, E.; PIZO, M. A.; MORELLATO, L. P. C. Polinização e dispersão de sementes em Myrtaceae do Brasil. Revista Brasileira de Botânica, São Paulo, v. 29, n. 4, p. 509-530, 2006.

HOWE, H. F.; SMALLWOOD, J. Ecology of seed dispersal. Annual Review of Ecology and Systematics, Palo Alto, v. 12, p. 201-228, 1982.

IBGE. Manual técnico da vegetação brasileira. Rio de Janeiro: IBGE, 1992. (Série Manuais Técnicos em Geociências).

JESUS, S.; MONTEIRO-FILHO, E. L. A. Frugivoria por aves em Schinus terebinthifolius (Anacardiaceae) e Myrsine coreacea (Myrsinaceae). Revista Brasileira de Ornitologia, São Paulo, v. 15, n. 4, p. 585-591, 2007.

JORDANO, P.; SCHUPP, E. W. Seed disperser effectiveness: the quantity component and patterns of seed rain for Prunus mahaleb. Ecological Monographs, Washington, v. 70, n. 4, p. 591-615, 2000.

JULIÁ, J. P.; PERIS, S. J. Do precipitation and food affect the reproduction of brown brocket deer Mazama gouazoubira (G. Fischer 1814) in conditions of semi-captivity? Anais da Academia Brasileira de Ciências, Rio de Janeiro, v. 82, n. 3, p. 629-635, 2010.

LAKE, J. C.; LEISHMAN, M. R. Invasion success of exotic plants in natural ecosystems: the role of disturbance, plant attributes and freedom from herbivores. Biological Conservation, Essex, v. 117, n. 2, p. 215-226, 2004.
LANDRUM, L. R.; KAWASAKI, M. L. The genera of Myrtaceae in Brazil: an illustrated synoptic treatment and identification keys. Brittonia, Bronx, v. 49, p. 508-536, 1997.

LANDRUM, L. R.; SHARP, W. P. Seed coat characters of some American Myrtinae (Myrtaceae): Psidium and related genera. Systematic Botany, Kent, v. 14, p. 370-376, 1989.

LEGENDRE, P.; LEGENDRE, L. Numerical ecology. Amsterdam: Elsevier, 2003.

MARCONDES-MACHADO, L. O.; ROSA, G. A. B. Frugivoria por aves em Cytharexyllum myrianthum Cham (Verbenaceae) em áreas de pastagens de Campinas, SP. Revista Brasileira de Ornitologia, São Paulo, v. 13, n. 1, p. 113-115, 2005.

MOTTA-JÚNIOR, J. C. Estrutura trófica e composição das avifaunas de três habitats terrestres na região central do Estado de São Paulo. Revista Brasileira de Ornitologia, São Leopoldo, v. 12, n. 2, p. 65-71, 1990.

NÓBREGA, A. M. F.; VALERI, S. V.; PAULA, R. C.; PAVANI, M. C. M. D.; SILVA, S. A. Banco de sementes de remanescentes naturais e de áreas reflorestadas em uma várzea do Rio Mogi-Guaçu - SP. Revista Árvore, Viçosa, v. 33, n. 3, p. 403-411, 2009.

ORWA, C.; MUTUA, A.; KINDT, R.; JAMNADASS, R.; SIMONS, A. Agroforestree Database: tree reference and selection guide version 4.0. World Agroforestry Centre, Kenya, 2009. Disponível em: $<$ http://www.worldagroforestry.org/af/treedb/>. Acesso em: 3 maio 2012.

PEREIRA, T. S.; ANDRADE, A. C. S. Germinação de Psidium guajava L. e Passiflora edulis Sims efeito da temperatura, substrato e morfologia do desenvolvimento pós-seminal. Revista Brasileira de Sementes, Londrina, v. 16, n. 1, p. 58-62, 1994.

PIZO, M. A. Seed dispersal and predation in two populations of Cabralea canjerana (Meliaceae) in the Atlantic forest of southeastern Brazil. Journal of Tropical Ecology, Cambridge, v. 13, p. 559-578, 1997. 
PIZO, M. A. The seed dispersers and fruit syndromes of Myrtaceae in Brazilian Atlantic forest. In: LEVEY, D. J.; SILVA, W. R.; GALETTI, M. Frugivores and seed dispersers: biodiversity and conservation perspectives. Wallingford: CABI Publishing, 2002, p. 129-143.

PIZO, M.A. Frugivory and habitat use by fruiteating birds in a fragmented landscape of southeast Brazil. Ornitologia Neotropical, Washington, v. 15, p. 117-126, 2004.

RICHARDSON, D. M.; ALLSOPP, N.; D’ANTONIO, C. M.; MILTON, S. J.; REJMÁNEK, M. Plant invasions - the role of mutualisms. Biological Reviews, Cambridge, v. 75, n. 1, p. 6593, 2000.

SCHUPP, E. W. Quantity, quality and effectiveness of seed dispersal by animals. Vegetatio, Dordrecht, v. 107/108, n. 1, p. 15-29, 1993.

SICK, H. Ornitologia brasileira. Rio de Janeiro: Nova Fronteira, 1997.

SIEGEL, S.; CASTELLAN JUNIOR, N. J. Nonparametric statistics for the behavioral sciences. New York: McGraw Hill, 1988.

SIGRIST, T. Aves do Brasil: uma visão artística. São Paulo: Fosfertil, 2006.

SILVA, J. M. C.; TABARELLI, M. Tree species impoverishment and the future flora of the Atlantic forest of Northeast Brazil. Nature, London, v. 404, p. 72-74. 2000.

SOMARRIBA, E. Effects of Livestock on seed germination of guava (Psidium guajava L.) Agroforestry Systems, Dordrecht, v. 4, n. 3, p. 233238, 1986.

STAGGEMEIER V. G.; GALETTI, M. Impacto humano afeta negativamente a dispersão de sementes de frutos ornitocóricos: uma perspectiva global. Revista Brasileira de Ornitologia, São Paulo, v. 15, n. 2, p. 281-287, 2007.
VOLPATO, G. H., MENDONÇA-LIMA, A. Estratégias de forrageamento: proposta de termos para a língua portuguesa. Revista Brasileira de Ornitologia, São Leopoldo, v. 10, n. 1, p. 101-105, 2002.

WILLIS, E. O. The composition of avian communities in remnant woodlots in southern Brazil. Papéis Avulsos de Zoologia, São Paulo, v. 33, n. 1, p. 1-25, 1979.

ZVIEJKOVSKI, I. P. Sucessão em uma pastagem tropical abandonada: mudanças estruturais e estimativa de recuperação florestal. 2008. 32 f. Dissertação (Mestrado em Ecologia de Ambientes Aquáticos Continentais) - Universidade Estadual de Maringá, Maringá, 2008. 
Silva, J. C. B. et al. 